\title{
杂质对氧化锆热障涂层性能的影响
}

\author{
冀晓鹃 ${ }^{1,2}$, 于月光 ${ }^{2}$, 卢晓亮 ${ }^{2}$ \\ (1. 东北大学 材料科学与工程学院, 沈阳 $110819 ; 2$. 北京矿冶科技集团有限公司, 北京 100160)
}

摘 要: 降低热障涂层面层中的低熔点杂质含量, 可提高涂层的高温稳定性和延长服役寿命。 $\mathrm{SiO}_{2} 、 \mathrm{Al}_{2} \mathrm{O}_{3}$ 和 $\mathrm{Fe}_{2} \mathrm{O}_{3}$ 是氧化钇稳定氧化锆(Yttria-Stabilized Zirconia, YSZ)热障涂层中几种常见的低熔点氧化物杂质, 均会对涂层的性能 产生一定的影响。本研究采用大气等离子喷涂法, 制备 $\mathrm{SiO}_{2} 、 \mathrm{Al}_{2} \mathrm{O}_{3}$ 和 $\mathrm{Fe}_{2} \mathrm{O}_{3}$ 的含量从小于 $0.01 \mathrm{wt} \%$ 增加至 $1.00 \mathrm{wt} \%$ 的 YSZ 热障涂层。采用扫描电镜(SEM)和透射电镜(TEM)研究了上述涂层的微观结构; 采用激光热导仪测试了涂层 的热扩散系数和抗热震次数。研究结果表明, 低熔点氧化物杂质对 YSZ 涂层的导热性、热处理状态的孔隙率具有明 显影响, 且更容易引起涂层的热震失效。当杂质氧化物含量在小于 $0.2 \mathrm{wt} \%$ 范围内变化时, 涂层的性能变化更为显著。 关 键 词: 热障涂层; 杂质; YSZ; 抗热震性能; 导热性

中图分类号: TG174 文献标识码: A

\section{Effects of Impurities on Properties of YSZ Thermal Barrier Coatings}

\author{
JI Xiaojuan ${ }^{1,2}$, YU Yueguang ${ }^{2}$, LU Xiaoliang ${ }^{2}$
}

(1. School of Materials Science and Engineering, Northeastern University, Shenyang 110819, China; 2. BGRIMM Technology Group, Beijing 100160, China)

\begin{abstract}
Reducing low-melting impurity content in thermal barrier coatings (TBCs) can improve the high temperature stability and service life of coatings. But there is rare quantitative study on the relationship between the low-melting impurity content and coating properties. In the present work, the effect of $\mathrm{SiO}_{2}, \mathrm{Al}_{2} \mathrm{O}_{3}$, and $\mathrm{Fe}_{2} \mathrm{O}_{3} \mathrm{im}$ purities on the properties of Yttria-Stabilized Zirconia (YSZ) TBCs prepared by atmospheric plasma spraying was investigated. The samples were prepared with the contents of these impurities being increased gradually from less than $0.01 \mathrm{wt} \%$ to $1.00 \mathrm{wt} \%$. The results demonstrated that those low-melting oxide impurities exhibit a significant effect on thermal conductivity and porosity of the coatings, playing a key role on thermal shock failure. The performance of coatings deteriorated especially significantly when the impurity oxide content is less than $0.2 \mathrm{wt} \%$.
\end{abstract}

Key words: thermal barrier coating; impurity; YSZ; thermal shock resistance; thermal conductivity

热障涂层(Thermal Barrier Coatings, TBCs)已在 发动机燃烧室、高压涡轮叶片等热端部件上得到广 泛应用。由于 TBCs 具有优异的隔热能力, 能够提 高部件工作温度, 并减少所需的冷却气量, 从而可 有效地提高发动机效率、减少排放、提高推重比 ${ }^{[1]}$ 。
目前应用最多的 TBCs 系统由金属粘结层和 YSZ 面 层组成, YSZ 因其导热系数低、在一定的工作条件 下稳定性高、热膨胀系数和韧性适宜, 是迄今为止 最成熟的 TBCs 面层材料。而不断提高热障涂层的 高温稳定性和使用寿命是研究的共同目标 ${ }^{[2]}$ 。

收稿日期: 2020-02-18; 收到修改稿日期：2020-03-01

基金项目: 国家科技支撑计划(2012BAE02B02)

National Key Technology Research and Development Program(2012BAE02B02)

作者简介: 冀晓鹃(1981-), 女, 博士研究生. E-mail: ji_xj@bgrimm.com JI Xiaojuan(1981-), female, PhD candidate. E-mail: ji_xj@bgrimm.com

通讯作者: 于月光, 教授. E-mail: yuyg@bgrimm.com YU Yueguang, professor. E-mail: yuyg@bgrimm.com 
通过改进热障涂层材料体系 ${ }^{[1,3-6]}$ 、优化涂层结

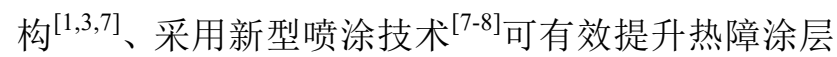
的性能。其中, 低杂质含量的高纯度热障涂层 ${ }^{[2,5,9-10]}$ 因具有更好的抗烧结性、更长的耐热冲击寿命而被 广泛关注。热障涂层的失效模式研究表明 ${ }^{[11-12]}$, TBCs 在服役过程中发生烧结, 将显著降低涂层的隔热效 果、应变容限及服役寿命 ${ }^{[13-15]}$ 。Vaßen 等 ${ }^{[16]}$ 指出, 在 $1400{ }^{\circ} \mathrm{C}$ 下保温 $20 \mathrm{~h}$, 高纯度 YSZ 涂层的烧结收缩 率低于含 $\mathrm{Al}_{2} \mathrm{O}_{3}$ 或 $\mathrm{SiO}_{2}$ 的 $\mathrm{YSZ}$ 涂层。当 $\mathrm{Al}_{2} \mathrm{O}_{3}$ 和 $\mathrm{SiO}_{2}$ 含量在 $0.02 \mathrm{wt} \% \sim 0.12 \mathrm{wt} \%$ 范围时, $\mathrm{YSZ}$ 涂层的 烧结收缩率与 $\mathrm{Al}_{2} \mathrm{O}_{3}$ 和 $\mathrm{SiO}_{2}$ 含量成线性关系。 $\mathrm{Paul}$ 等 ${ }^{[17]}$ 研究表明, 将高纯度 YSZ 和含有 $\mathrm{Al}_{2} \mathrm{O}_{3} 、 \mathrm{SiO}_{2}$ 杂质的 YSZ 涂层在 $1400{ }^{\circ} \mathrm{C}$ 下进行热处理, 当杂 质含量从 $0.1 \mathrm{wt} \% \sim 0.2 \mathrm{wt} \%$ 下降到 $0.01 \mathrm{wt} \% \sim 0.05 \mathrm{wt} \%$ 时, 涂层的烧结速率显著降低。Xie 等 ${ }^{[18]}$ 提出杂质 含量的增加会加速相的分解。Helminiak 等 ${ }^{[19]}$ 也认 为, 高纯度 YSZ 涂层的相变速率显著低于杂质含量 高的涂层。然而以上研究中的杂质含量无可比性, 仅说明当 $\mathrm{YSZ}$ 涂层中存在 $\mathrm{SiO}_{2}$ 或 $\mathrm{Al}_{2} \mathrm{O}_{3}$ 杂质时显著 影响涂层的性能, 但对杂质与涂层性能的定量关系 尚未给出规律性的说明, 对杂质含量增加或减少时 涂层性能的变化未做深入研究。因此, 只有明确杂 质含量与涂层性能之间的定量或半定量关系, 并给 出杂质含量的控制极限, 才有助于 YSZ 涂层材料及 涂层的成分设计和杂质含量的控制。

本研究设计了系列含低熔点氧化物杂质 $\mathrm{SiO}_{2}$ 、 $\mathrm{Al}_{2} \mathrm{O}_{3} 、 \mathrm{Fe}_{2} \mathrm{O}_{3}$ 的 $\mathrm{YSZ}$ 热障涂层, 杂质含量范围为小 于 $0.01 \mathrm{wt} \% \sim 1.00 \mathrm{wt} \%$ 。研究了系列涂层的抗热震性、 等温时效后的微观结构变化和隔热性能, 并探究了 杂质对涂层性能的影响机制。通过研究杂质含量与 YSZ 涂层性能之间的关系给出控制杂质含量极限的 建议。

\section{1 实验方法}

\section{1 制备喷涂粉末材料}

采用喷雾干燥一烧结方法制备 YSZ 粉末。将高 纯度纳米级 YSZ 粉末(化学成分见表 1)与杂质氧化 物纳米粉末(纯度 $>99.9 \mathrm{wt} \%$ ) 按设计比例(表 2)进行湿 法球磨 $24 \mathrm{~h}$ 。YSZ 粉末的平均粒径为 $60 \mathrm{~nm}$, 氧化 物粉末的平均粒径为 $30 \mathrm{~nm}$, 团聚 YSZ 粉末的粒度 范围为 $25 \sim 63 \mu \mathrm{m}$ 。

\section{2 制备涂层}

采用超音速火焰喷涂 (High-Velocity OxygenFuel, HVOF)制备 $\mathrm{Ni}_{23} \mathrm{Co}_{25} \mathrm{Cr}_{6} \mathrm{~A}_{10.6} \mathrm{Y}$ 粘结层, 设计厚 度 $100 \mu \mathrm{m}$, 工艺参数见表 3 。采用大气等离子喷涂
(Air Plasma Spraying, APS)制备面层, 厚度为 250 $300 \mu \mathrm{m}$, 工艺参数见表 4 。由 APS 制备用于测试热扩 散系数和进行高温热处理的单层 YSZ 涂层, 厚度为 1.00 1.25 mm。采用 KSL-1700X 型电炉进行涂层热 处理试验, 以 $5{ }^{\circ} \mathrm{C} / \mathrm{min}$ 的速度升温至保温温度, 保温 一定时间后随炉冷却。YSZ 面层中杂质的实测成分 如表 2 所示, 可以看出, 涂层中杂质含量较设计值有 所降低, 这是由于制备粉末过程中产生的误差及喷 涂过程中的烧损造成的。涂层中的杂质含量基本处 于同一数量级水平, 因此可采用设计成分进行对比。

表 1 YSZ 原料的化学成分 $(\mathrm{wt} \%)$

Table 1 Chemical composition of YSZ raw materials (wt\%)

\begin{tabular}{cccccc}
\hline $\mathrm{ZrO}_{2}$ & $\mathrm{HfO}_{2}$ & $\mathrm{TiO}_{2}$ & $\mathrm{SiO}_{2}$ & $\mathrm{MgO}$ & $\mathrm{Fe}_{2} \mathrm{O}_{3}$ \\
\hline 90.28 & 1.55 & $<0.01$ & $<0.01$ & $<0.01$ & $<0.01$ \\
\hline \hline $\mathrm{Y}_{2} \mathrm{O}_{3}$ & $\mathrm{Al}_{2} \mathrm{O}_{3}$ & $\mathrm{CaO}$ & $\mathrm{Na}_{2} \mathrm{O}$ & $\mathrm{K}_{2} \mathrm{O}$ & \\
\hline 7.92 & 0.011 & $<0.01$ & $<0.01$ & $<0.01$ & \\
\hline
\end{tabular}

表 2 YSZ 喷涂粉末的设计成分 $(D)$ 及涂层 的实测成分 $(M)(w t \%)$

Table 2 Design composition (D) of YSZ powders and measured composition $(M)$ of YSZ coatings (wt\%)

\begin{tabular}{|c|c|c|c|c|c|c|c|c|}
\hline \multirow{2}{*}{$\mathrm{No}$} & \multicolumn{2}{|c|}{$\mathrm{SiO}_{2}$} & \multirow{2}{*}{ To. } & \multicolumn{2}{|c|}{$\mathrm{Al}_{2} \mathrm{O}_{3}$} & \multirow{2}{*}{ No. } & \multicolumn{2}{|c|}{$\mathrm{Fe}_{2} \mathrm{O}_{3}$} \\
\hline & $D$ & $M$ & & $D$ & $M$ & & $D$ & $M$ \\
\hline HP & $<0.01$ & $<0.01$ & - & $<0.01$ & $<0.01$ & - & $<0.01$ & 0.0049 \\
\hline $\mathrm{S} 1$ & 0.02 & 0.013 & A1 & 0.02 & 0.014 & F1 & 0.02 & 0.0140 \\
\hline $\mathrm{S} 2$ & 0.06 & 0.038 & A2 & 0.06 & 0.030 & F2 & 0.06 & 0.0390 \\
\hline $\mathrm{S} 3$ & 0.10 & 0.064 & A3 & 0.10 & 0.051 & F3 & 0.10 & 0.0800 \\
\hline S4 & 0.16 & 0.110 & A4 & 0.15 & 0.079 & F4 & 0.16 & 0.1100 \\
\hline S5 & 0.20 & 0.150 & A5 & 0.20 & 0.120 & F5 & 0.20 & 0.1400 \\
\hline S6 & 0.36 & 0.260 & A6 & 0.35 & 0.230 & F6 & 0.36 & 0.3000 \\
\hline S7 & 0.50 & 0.320 & A7 & 0.50 & 0.300 & F7 & 0.50 & 0.3400 \\
\hline S8 & 0.66 & 0.430 & A8 & 0.65 & 0.380 & F8 & 0.66 & 0.4600 \\
\hline S9 & 0.80 & 0.550 & A9 & 0.80 & 0.450 & F9 & 0.80 & 0.5700 \\
\hline $\mathrm{S} 10$ & 1.00 & 0.620 & A 10 & 1.00 & 0.640 & F10 & 1.00 & 0.5900 \\
\hline
\end{tabular}

表 3 HVOF 喷涂工艺参数

Table 3 Parameters of HVOF process

\begin{tabular}{|c|c|c|c|c|}
\hline $\begin{array}{l}\text { Coal oil } \\
/\left(\mathrm{L} \cdot \mathrm{h}^{-1}\right)\end{array}$ & $\frac{\mathrm{O}_{2}}{/\left(\mathrm{L} \cdot \mathrm{min}^{-1}\right)}$ & $\begin{array}{c}\text { Ar (Carrier gas) } \\
\quad /\left(\mathrm{L} \cdot \mathrm{min}^{-1}\right)\end{array}$ & $\begin{array}{l}\text { Powder feeding } \\
\text { rate } /\left(\mathrm{g} \cdot \mathrm{min}^{-1}\right)\end{array}$ & $\begin{array}{c}\text { Distance/ } \\
\mathrm{mm}\end{array}$ \\
\hline 26 & 900 & 8 & 75 & 380 \\
\hline
\end{tabular}

表 4 APS 喷涂工艺参数

Table 4 Parameters of APS process

\begin{tabular}{cccc}
\hline Current/A & Voltage/V & Power $/ \mathrm{kW}$ & Distance $/ \mathrm{mm}$ \\
\hline 620 & 76 & 47 & 100 \\
\hline $\mathrm{Ar} /\left(\mathrm{L} \cdot \mathrm{min}^{-1}\right)$ & $\mathrm{H}_{2} /\left(\mathrm{L} \cdot \mathrm{min}^{-1}\right)$ & $\begin{array}{c}\text { Ar(Carrier gas }) / \\
\left(\mathrm{L} \cdot \mathrm{min}^{-1}\right)\end{array}$ & $\begin{array}{c}\text { Powder feeding } \\
\text { rate } /\left(\mathrm{g} \cdot \mathrm{min}^{-1}\right)\end{array}$ \\
\hline 38 & 13 & 4.5 & 30 \\
\hline
\end{tabular}




\section{3 微观结构表征及性能测试}

采用日立 SU5000 型扫描电镜分析形貌, 采用日 本电子 JEM2100 型透射电镜分析结构。根据 ASTM E 2109 B 测试孔隙率。采用 Netzsch LFA 427 型激光 热导仪测量 YSZ 涂层的热扩散系数, 测试方法参照 ASTM E1461。测量前, 将涂层加热到 $1400{ }^{\circ} \mathrm{C}$ 保温 $2 \mathrm{~h}$ (升温速度 $5{ }^{\circ} \mathrm{C} / \mathrm{min}$ ) 后随炉冷却。

涂层抗热震性能的测试过程如下: 将涂层试样 置入 $1100{ }^{\circ} \mathrm{C}$ 箱式电炉保温 $10 \mathrm{~min}$ 后, 取出投入约 $25{ }^{\circ} \mathrm{C}$ 的纯净水中保持 $60 \mathrm{~s}$, 如此反复循环, 直至涂 层剥落面积达到总面积的 $5 \%$ 时视为涂层热震失效。

\section{2 结果与讨论}

\section{1 显微形貌及孔隙率}

图 1 为 $\mathrm{S} 1$ 样品(见表 2)的喷涂态涂层横截面的 典型微观形貌, 可以看出 YSZ 面层为典型的层状结 构, 夹杂部分未熔粉末的微细颗粒。

对不同杂质含量的 YSZ 涂层在 $1400{ }^{\circ} \mathrm{C}$ 下保温 $100 \mathrm{~h}$ 后的孔隙率进行对比, 如图 2 所示。涂层经热 处理后孔隙率均随氧化物杂质含量的增加而降低。 当杂质含量低于 $0.01 \mathrm{wt} \%$ 时, 涂层的孔隙率均在 $6.5 \% \sim 7.5 \%$ 范围内。当 $\mathrm{Al}_{2} \mathrm{O}_{3}$ 含量从 $0.01 \mathrm{wt} \%$ 增加至 $0.10 \mathrm{wt} \% 、 \mathrm{SiO}_{2}$ 和 $\mathrm{Fe}_{2} \mathrm{O}_{3}$ 含量从 $0.01 \mathrm{wt} \%$ 增加至 $0.20 \mathrm{wt} \%$ 时, 涂层孔隙率显著降低, 分别降低了 $0.733 \% 、 1.053 \%$ 和 $1.500 \%$ 。当以上杂质含量继续增 加至 $0.65 \mathrm{wt} \%$, 即含量增加了 $0.45 \mathrm{wt} \% \sim 0.55 \mathrm{wt} \%$ 时, 孔隙率降幅减缓, 分别仅降低了 $0.694 \% 、 0.537 \%$ 和 $1.15 \%$ 。杂质含量继续增加至 $1.00 \mathrm{wt} \%$ 时, 含 $\mathrm{SiO}_{2}$ 涂层的孔隙率下降相对显著, 降低了 $1.749 \%$ 。说明 在高温条件下, 杂质含量在较低水平范围内变化时, $\mathrm{Al}_{2} \mathrm{O}_{3}$ 含量在小于 $0.01 \mathrm{wt} \% \sim 0.10 \mathrm{wt} \%$ 范围内、 $\mathrm{SiO}_{2}$ 和 $\mathrm{Fe}_{2} \mathrm{O}_{3}$ 含量在小于 $0.01 \mathrm{wt} \% \sim 0.20 \mathrm{wt} \%$ 范围内时, 涂层孔隙率显著降低。在杂质含量相对较高时, $\mathrm{SiO}_{2}$ 对涂层孔隙率的影响较为显著。

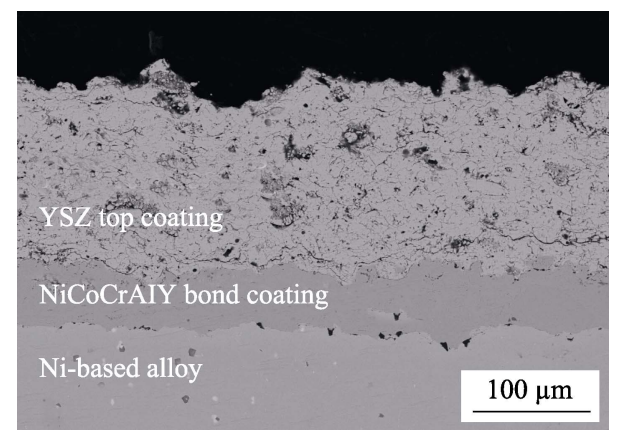

图 $1 \mathrm{~S} 1$ 双层热障涂层的显微形貌

Fig. 1 Microstructure of S1 two-layer thermal barrier coaing

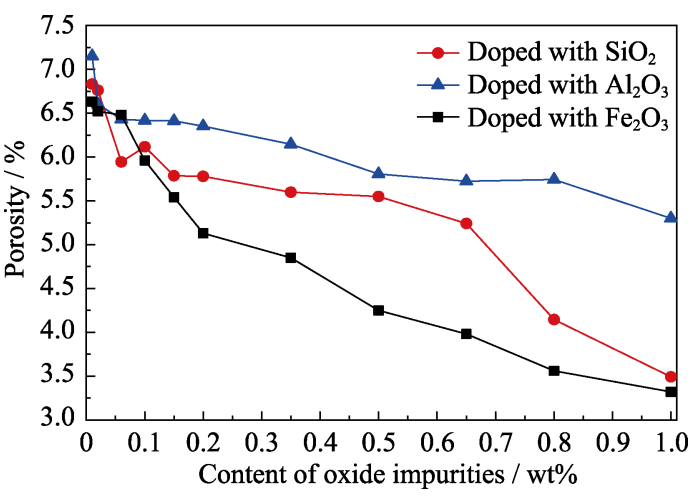

图 $21400{ }^{\circ} \mathrm{C}$ 热处理 $100 \mathrm{~h}$ 后 YSZ 涂层的孔隙率

Fig. 2 Porosities of YSZ coatings heat-treated at $1400{ }^{\circ} \mathrm{C}$ for $100 \mathrm{~h}$

为了比较不同氧化物杂质在高温下促进 YSZ 涂层致密化行为的差异, 对杂质含量均为 $1 \mathrm{wt} \%$ 的 涂层, 在不同温度和不同保温时间下进行热处理, 涂层的孔隙率如图 3 所示。

从图 3 可以看出, 高纯度涂层的孔隙率随热处 理温度的升高和热处理时间的延长发生的变化率最 小。含 $\mathrm{SiO}_{2}$ 杂质 YSZ 涂层的孔隙率随热处理温度 的升高和热处理时间的延长显著降低, 表明该涂层 具有更明显的烧结性 ${ }^{[16]}$ 。含 $\mathrm{Al}_{2} \mathrm{O}_{3}$ 杂质 YSZ 涂层的 孔隙率与之相似, 但是下降幅度较为平缓。含 $\mathrm{Fe}_{2} \mathrm{O}_{3}$ 杂质 YSZ 涂层的孔隙率在不同温度和保温时间条 件下波动明显, 且对热处理时间更为敏感。涂层孔 隙率可表征涂层在高温下的烧结状态 ${ }^{[18]}$, 以上结果 表明, $\mathrm{SiO}_{2}$ 和 $\mathrm{Fe}_{2} \mathrm{O}_{3}$ 对涂层使用温度和使用寿命产 生影响。

\section{2 热扩散系数}

不同杂质含量的 YSZ 涂层在 $1200{ }^{\circ} \mathrm{C}$ 的热扩散 系数如图 4 所示。热扩散系数是反映涂层隔热性能 的重要参量。由图 4 可知, YSZ 涂层的热扩散系

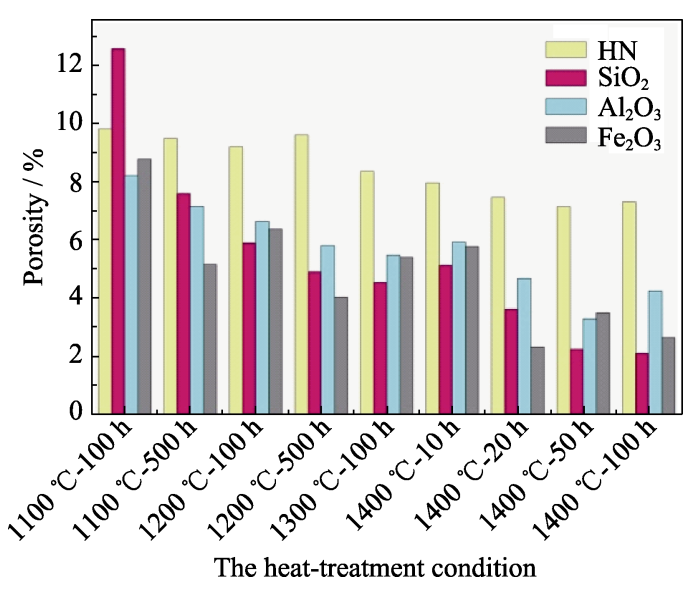

图 3 不同热处理条件下含 $1 \mathrm{wt} \%$ 氧化物 YSZ 涂层的孔隙率 Fig. 3 Porosities of YSZ coatings doped with $1 \mathrm{wt} \%$ oxides after heat treatment 
数随 $\mathrm{SiO}_{2} 、 \mathrm{Fe}_{2} \mathrm{O}_{3}$ 含量的增加而增大。这与高温处 理后不同 $\mathrm{SiO}_{2} 、 \mathrm{Fe}_{2} \mathrm{O}_{3}$ 含量的 YSZ 涂层的烧结状态 有关。

涂层的热扩散系数通常是涂层固有导热性和微 观结构(如涂层孔隙率)的共同效应 ${ }^{[20-21]}$ 。根据 Wang 等 ${ }^{[22]}$ 的研究结果, 涂层中孔隙率对传热的影响最 大。涂层中存在的微裂纹和微气孔会大大降低涂层 的热扩散系数, 提高涂层隔热性能 ${ }^{[21]}$ 。而涂层热扩 散系数的增大主要是由于 $1400{ }^{\circ} \mathrm{C}$ 下 $\mathrm{SiO}_{2} 、 \mathrm{Fe}_{2} \mathrm{O}_{3}$ 促进了涂层的烧结和致密化 ${ }^{[23-24]}$ 。根据涂层孔隙率 的结果, 随着 $\mathrm{SiO}_{2} 、 \mathrm{Fe}_{2} \mathrm{O}_{3}$ 含量的增加, 孔隙率降低, 涂层更加致密, 导致涂层热扩散系数增大。随杂质 含量的增加, 含 $\mathrm{SiO}_{2}$ 涂层的热扩散系数变化率大, 这与高温处理后含 $\mathrm{SiO}_{2}$ 涂层较大的孔隙率变化率 相一致。而含有 $\mathrm{Al}_{2} \mathrm{O}_{3}$ 的 YSZ 涂层热扩散系数随 $\mathrm{Al}_{2} \mathrm{O}_{3}$ 含量增加表现出先降低后升高的趋势。当在 $\mathrm{YSZ}$ 中加入 $\mathrm{Al}_{2} \mathrm{O}_{3}$ 时, 由于 $\mathrm{Al}^{3+}$ 比 $\mathrm{Y}^{3+}$ 和 $\mathrm{Zr}^{4+}$ 轻, 这 导致在 YSZ 的 $\mathrm{Y}^{3+}$ 或 $\mathrm{Zr}^{4+}$ 位置处由 $\mathrm{Al}^{3+}$ 取代产生质 量缺陷, 这个缺陷会造成一个声子散射中心, 增强 材料的隔热性能 ${ }^{[24]}$ 。当 $\mathrm{Al}_{2} \mathrm{O}_{3}$ 含量增加到一定程度 时, 由于加入到涂层中的杂质促进了烧结, 提高了 涂层的致密度 ${ }^{[18]}$, 而这种效应对高温下涂层的热性 能起着更重要的作用 ${ }^{[20]}$, 进而引起涂层热扩散系数 的升高。

\section{3 热震寿命}

含有 $\mathrm{SiO}_{2} 、 \mathrm{Al}_{2} \mathrm{O}_{3} 、 \mathrm{Fe}_{2} \mathrm{O}_{3}$ 杂质的 $\mathrm{YSZ}$ 涂层平均 热震寿命如图 5 所示。当杂质含量均小于 $0.01 \mathrm{wt} \%$ 时, 涂层热震寿命均约为 130 次。随着杂质含量的 增加, 热震寿命均呈下降趋势。

当杂质含量从小于 $0.01 \mathrm{wt} \%$ 增加至 $0.02 \mathrm{wt} \%$ 时, 涂层热震寿命降幅明显。 $\mathrm{SiO}_{2} 、 \mathrm{Fe}_{2} \mathrm{O}_{3}$ 含量增加至 $0.10 \mathrm{wt} \%$ 以上时涂层热震寿命降幅减缓。同样的现 象也发生在 $\mathrm{Al}_{2} \mathrm{O}_{3}{ }^{[25]}$ 含量达到 $0.2 \mathrm{wt} \%$ 以上时, 涂层 热震寿命降幅减缓。低熔点杂质会缩短 YSZ 涂层的

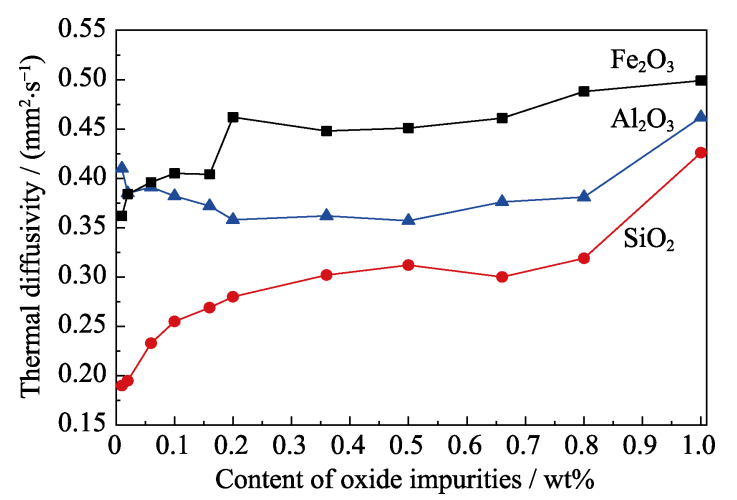

图 4 YSZ 涂层的热扩散系数

Fig. 4 Thermal diffusivity of YSZ coatings

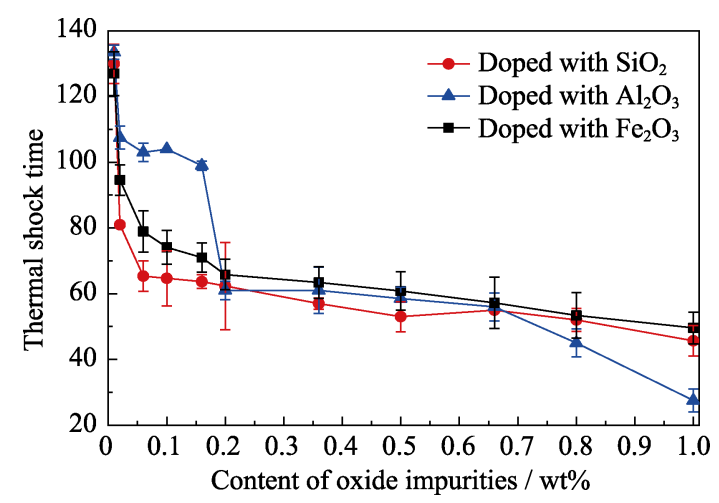

图 5 YSZ 涂层的热震寿命

Fig. 5 Thermal shock lifetime of YSZ coatings

热震寿命，这与 Xie 等 ${ }^{[18]}$ 对比高纯度与含杂质的 YSZ 涂层热循环寿命结果一致。同时, 杂质含量越低时, 杂质含量的变化对 YSZ 涂层的热震寿命影响越显 著。这与本研究中涂层在高温条件下的孔隙率变化 情况一致。当 $\mathrm{Al}_{2} \mathrm{O}_{3}$ 含量在 $0.02 \mathrm{wt} \% \sim 0.15 \mathrm{wt} \%$ 之间 时, 涂层热震寿命下降缓慢, 热震次数变化出现一 个范围较窄的平台, 随后当 $\mathrm{Al}_{2} \mathrm{O}_{3}$ 含量继续增加至 $0.2 \mathrm{wt} \%$ 时, 涂层热震寿命显著降低, 这是由于原料 $\mathrm{Al}_{2} \mathrm{O}_{3}$ 粉末有团聚现象, 未能均匀、分散地填充至 YSZ 体系中, 未起到促进离子扩散的作用。

\section{4 透射电镜形貌}

采用透射电镜观察了 S10、A10 涂层在热处理 前后的微观组织变化, 如图 6 和图 7 所示, 分别为 S10、A 10 涂层喷涂态及经过 $1400{ }^{\circ} \mathrm{C} / 10 \mathrm{~h}$ 热处理 后涂层的透射电镜照片及衍射斑点。

如图 6 所示, S10 涂层喷涂态的晶粒大小约为 200 300 nm, 热处理后晶粒明显长大, 约为 600 $800 \mathrm{~nm}$ 。图 6(b)中间狭长白亮区域为非晶态 $\mathrm{SiO}_{2}$, 在晶界处富集并在高温形成玻璃相, 使晶界变得圆

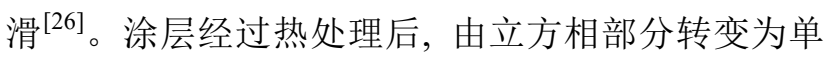
斜相, 通过计算发现, 立方相晶格参数由喷涂态时 的 $0.5115 \mathrm{~nm}$ 缩小至热处理后的 $0.5057 \mathrm{~nm}$, 说明部 分 $\mathrm{SiO}_{2}$ 进入 $\mathrm{ZrO}_{2}$ 晶格中, 由于 $\mathrm{Si}$ 的原子半径较小, 使晶格发生畸变。而这种含有 $\mathrm{Si} 、 \mathrm{Y} 、 \mathrm{Zr}$ 的共晶化 合物熔点较低, 这也是加速涂层在高温下烧结的 原因 ${ }^{[27]}$ 。

如图 7 所示, $\mathrm{A} 10$ 涂层喷涂态的晶粒大小约为 $200 \mathrm{~nm}$, 这与含 $\mathrm{SiO}_{2}$ 的涂层类似, 而热处理后晶粒 明显长大, 约为 $600 \mathrm{~nm} \sim 1 \mu \mathrm{m}$ 。文献表明 ${ }^{[28-29]}$, YSZ 体系中晶粒长大过程受阳离子扩散过程的影响, 部 分 $\mathrm{Al}^{3+}$ 离子替代 $\mathrm{Zr}^{4+}$ 离子, 为了维持电荷平衡, 阳 离子空位浓度及间隙浓度增加, 而间隙浓度为氧化 锆中扩散速率的控制环节, 进而促进晶粒长大, 并 改善了固相烧结 ${ }^{[30]}$ 。 

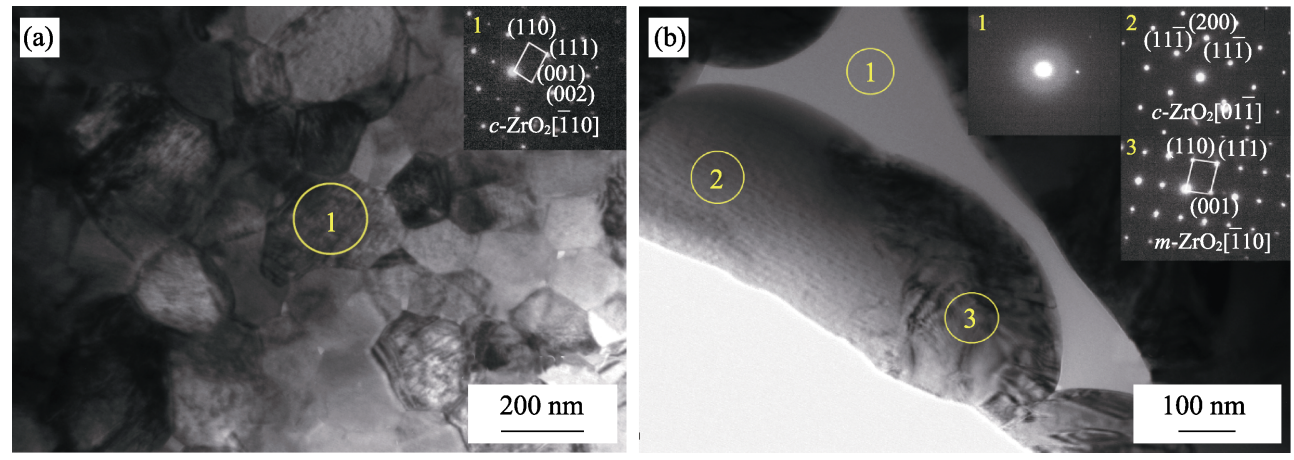

图 6 含 $1 \mathrm{wt} \% \mathrm{SiO}_{2} \mathrm{YSZ}$ 涂层的(a)喷涂态及(b)经 $1400{ }^{\circ} \mathrm{C}$ 热处理 $10 \mathrm{~h}$ 后的 $\mathrm{TEM}$ 照片及衍射斑点

Fig. 6 TEM microstructures and diffraction spots of YSZ coatings doped with $1 \mathrm{wt} \% \mathrm{SiO}_{2}$ (a) As-sprayed; (b) After heat- treatment at $1400{ }^{\circ} \mathrm{C}$ for $10 \mathrm{~h}$
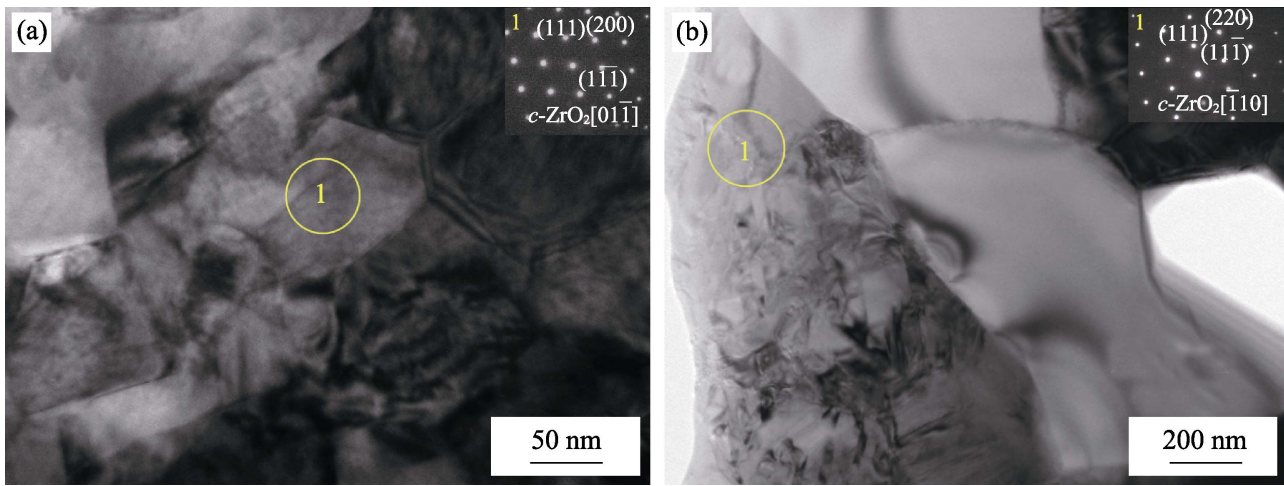

图 7 含 $1 \mathrm{wt} \% \mathrm{Al}_{2} \mathrm{O}_{3}$ 的 $\mathrm{YSZ}$ 涂层的(a)喷涂态及(b)经 $1400{ }^{\circ} \mathrm{C}$ 热处理 $10 \mathrm{~h}$ 后的 $\mathrm{TEM}$ 照片及衍射斑点

Fig. 7 TEM microstructure and diffraction spots of YSZ coatings doped with $1 \mathrm{wt} \% \mathrm{Al}_{2} \mathrm{O}_{3}$ (a) As-sprayed; (b) After heat- treatment at $1400{ }^{\circ} \mathrm{C}$ for $10 \mathrm{~h}$

\section{3 结论}

本研究设计制备了分别含有(小于 $1.00 \mathrm{wt} \%) \mathrm{SiO}_{2}$ 、 $\mathrm{Al}_{2} \mathrm{O}_{3} 、 \mathrm{Fe}_{2} \mathrm{O}_{3}$ 低熔点氧化物的 YSZ 热障涂层, 并对 涂层的微观形貌、抗热震性能和陶瓷层的导热性能 进行了分析，就低熔点氧化物对 YSZ 热障涂层性能 的影响得出以下结论:

1) 对 YSZ 陶瓷面层经过不同条件热处理后的 截面孔隙率的分析发现，随着低熔点氧化物杂质含 量的增加，高温下涂层孔隙率下降明显, $\mathrm{SiO}_{2}$ 对涂 层服役温度和时间均敏感, $\mathrm{Fe}_{2} \mathrm{O}_{3}$ 对涂层服役时间更 敏感, $\mathrm{SiO}_{2}$ 和 $\mathrm{Fe}_{2} \mathrm{O}_{3}$ 易对涂层的使用温度和服役寿 命产生不利影响。

2) 低熔点氧化物影响涂层的烧结程度，从而影 响涂层的导热性。

3) 随着低熔点氧化物含量的增加, 涂层的热震 寿命缩短。综合考虑氧化物杂质对涂层微观形貌及 热震性能的影响，在 YSZ 热障涂层中，控制 $\mathrm{SiO}_{2}$ 、 $\mathrm{Fe}_{2} \mathrm{O}_{3} 、 \mathrm{Al}_{2} \mathrm{O}_{3}$ 的含量在 $0.10 \mathrm{wt} \%$ 以下，尤其是控制在 $0.01 \mathrm{wt} \%$ 以下时，涂层抗热震性及隔热性能更优异。
参考文献:

[1] DAROLIA R. Thermal barrier coatings technology: critical review, progress update, remaining challenges and prospects. International Materials Reviews, 2013, 58(6): 315-348.

[2] CURRY N, MARKOCSAN N, LI X H, et al. Next generation thermal barrier coatings for the gas turbine industry. Journal of Thermal Spray Technology, 2011, 20(1/2): 108-115.

[3] STÖVER D, PRACHT G, LEHMANN H, et al. New material concepts for the next generation of plasma-sprayed thermal barrier coatings. Journal of Thermal Spray Technology, 2004, 13(1): 76-83.

[4] XUE Z L, GUO H B, GONG S K, et al. Novel ceramic materials for thermal barrier coatings. Journal of Aeronautical Materials, 2018, 38(2): 10-20.

[5] CURRY N, JANIKOWSKI W, PALA Z, et al. Impact of impurity content on the sintering resistance and phase stability of dysprosiaand yttria-stabilized zirconia thermal barrier coatings. Journal of Thermal Spray Technology, 2014, 23(1/2): 160-169.

[6] LYU G, CHOI B G, LU Z, et al. Effect of thermal cycling frequency on the durability of Yb-Gd-Y-based thermal barrier coatings. Surface \& Coatings Technology, 2019, 364: 187-195.

[7] GORAL M, KOTOWSKI S, NOWOTNIK A, et al. PS-PVD deposition of thermal barrier coatings. Surface \& Coatings Technology, 2013, 237: 51-55.

[8] LATKA L. Thermal barrier coatings manufactured by suspension plasma spraying- a review. Advances in Materials Science, 2018, 18(3): 95-117. 
[9] JONNALAGADDA K P, ERIKSSON R, LI X H, et al. Thermal barrier coatings: life model development and validation. Surface \& Coatings Technology, 2019, 362: 293-301.

[10] PARK H M, JUN S H, LYU G, et al. Thermal durability of thermal barrier coatings in furnace cyclic thermal fatigue test: effects of purity and monoclinic phase in feedstock powder. Journal of the Korean Ceramic Society, 2018, 55(6): 608-617.

[11] KARLSSON A M. Modeling failures of thermal barrier coatings. Key Engineering Materials, 2007, 333: 155-166.

[12] HUA J J, ZHANG L P, LIU Z W, et al. Progress of research on the failure mechanism of thermal barrier coatings. Journal of Inorganic Materials, 2012, 27(7): 681-686.

[13] MATSUI K. Sintering kinetics at constant rates of heating: mechanism of silica-enhanced sintering of fine zirconia powder. Journal of the American Ceramic Society, 2008, 91(8): 2534-2539.

[14] TSIPAS S A, GOLOSNOY I O, DAMANI R, et al. The effect of a high thermal gradient on sintering and stiffening in the top coat of a thermal barrier coating system. Journal of Thermal Spray Technology, 2004, 13(3): 370-376.

[15] CHOI S R, ZHU D M, MILLER R A. Effect of sintering on mechanical properties of plasma-sprayed zirconia-based thermal barrier coatings. Journal of the American Ceramic Society, 2005, 88(10): 2859-2867.

[16] VAßEN R, CZECH N, MALLÉNER W, et al. Influence of impurity content and porosity of plasma-sprayed yttria-stabilized zirconia layers on the sintering behaviour. Surface and Coatings Technology, 2001, 141: 135-140.

[17] PAUL S, CIPITRIA A, GOLOSNOY I O, et al. Effects of impurity content on the sintering characteristics of plasma-sprayed zirconia. Journal of Thermal Spray Technology, 2007, 16(5/6): 798-803.

[18] XIE L, DORFMAN M R, CIPITRIA A, et al. Properties and performance of high-purity thermal barrier coatings. Journal of Thermal Spray Technology, 2007, 16(5/6): 804-808.

[19] HELMINIAK M A, YANAR N M, PETTIT F S, et al. The behavior of high-purity, low-density air plasma sprayed thermal barrier coatings. Surface \& Coatings Technology, 2009, 204: 793-796.
[20] MARKOCSAN N, NYLÉN P, WIGREN J, et al. Low thermal conductivity coatings for gas turbine applications. Journal of Thermal Spray Technology, 2007, 16(4): 498-505.

[21] ZHU D M, MILLER R A. Development of advanced low conductivity thermal barrier coatings. International Journal of Applied Ceramic Technology, 2004, 1(1): 86-94.

[22] WANG L, WANG Y, SUN X G, et al. Influence of pores on the thermal insulation behavior of thermal barrier coatings prepared by atmospheric plasma spray. Materials and Design, 2011, 32: 36-47.

[23] ZHU D M, MILLER R A. Thermal conductivity and elastic modulus evolution of thermal barrier coatings under high heat flux conditions. Journal of Thermal Spray Technology, 2000, 9(2): $175-180$.

[24] ZHU D M, MILLER R A, NAGARAJ B A, et al. Thermal conductivity of EB-PVD thermal barrier coatings evaluated by a steadystate laser heat flux technique. Surface and Coatings Technology, 2001, 138: $1-8$.

[25] LI Y J, YU Y G, JI X J, et al. Effects of $\mathrm{Al}_{2} \mathrm{O}_{3}$ content on properties of YSZ thermal barrier coatings. Thermal Spray Technology, 2018, 10(1): 61-67.

[26] GREMILLARD L, EPICIER T, CHEVALIER J, et al. Microstructural stucy of silica-doped zirconia ceramics. Acta Materialia, 2000, 48: $4647-4652$.

[27] HODGSON S N B, CAWLEY J, CLUBLEY M. The role of $\mathrm{SiO}_{2}$ impurities in the microstructure and properties of Y-TZP. Journal of Materials Processing Technology, 1999, 86: 139-145.

[28] MATSUI K, YOSHIDA H, IKUHARA Y. Phase-transformation and grain-growth kinetics in yttria-stabilized tetragonal zirconia polycrystal doped with a small amount of alumina. Journal of the European Ceramic Society, 2010, 30: 1679-1690.

[29] SAKKA Y, ISHII T, SUZUKI T S, et al. Fabrication of high-strain rate superplastic yttria-doped zirconia polycrystals by adding manganese and aluminum oxides. Journal of the European Ceramic Society, 2004, 24: 449-453.

[30] WU S X, BROOK R J. Kinetics of densification in stabilized zirconia. Solid State Ionics, 1984, 14: 123-130. 